\title{
Vertical Transfer of Momentum by Inertia-Gravity Internal Waves on a Two-Dimensional Shear Flow
}

\author{
A. A. Slepyshev \\ Marine Hydrophysical Institute of RAS, Sevastopol, Russian Federation \\ ⿶slep55@mail.ru
}

Purpose. The paper is aimed at investigating the momentum vertical transfer by inertia-gravity internal waves on a two-dimensional flow with a vertical shear of velocity, and also at studying the Stokes drift of liquid particles and the mean current effect on it.

Methods and Results. Free internal waves in an infinite basin of constant depth are considered in the Boussinesq approximation with the regard for the Earth rotation. Two components of the mean current velocity depend on the vertical coordinate. The equation for the vertical velocity amplitude has complex coefficients; therefore the eigenfunction and the wave frequency are complex. The corresponding boundary value problem is solved numerically by the implicit Adams scheme of the third order of accuracy. The wave frequency at a fixed wavenumber was found by the shooting method. It was determined that the frequency imaginary part was small and could be either negative or positive depending on a wave number and a mode number. Thus, both weak attenuation and weak amplification of an internal wave are possible. The vertical wave momentum fluxes are nonzero and can exceed the corresponding turbulent fluxes. The Stokes drift velocity, transverse to the wave direction, is nonzero and less than the longitudinal velocity. The vertical component of the Stokes drift velocity is also nonzero and four orders of magnitude less than the longitudinal component. The signs of the vertical component of the Stokes drift velocity for the waves with the frequencies 10 and $16 \mathrm{cph}$ are opposite, since the signs of their frequency imaginary parts are different; and the vertical component of the Stokes drift velocity is proportional to the wave frequency imaginary part.

Conclusions. The vertical momentum wave flux of inertia-gravity internal waves differs from zero in the presence of the current whose velocity component, transverse to the wave propagation direction, depends on the vertical coordinate. The component of the Stokes drift velocity, transverse to the wave propagation direction, is nonzero and less than the longitudinal one. The vertical component of the Stokes drift velocity is also nonzero and can contribute to formation of the vertical fine structure.

Keywords: internal waves, imaginary correction to frequency, wave momentum flux, Stokes drift

Acknowledgements: the author is grateful to N.O. Ankudinov for numerical calculations in solving the boundary problem. The study was carried out within the framework of the state task on theme No. 0827-2019-0003 "Fundamental investigations of oceanological processes conditioning state and evolution of marine environment being affected by natural and anthropogenic factors, based on the observation and modeling methods".

For citation: Slepyshev, A.A., 2021. Vertical Transfer of Momentum by Inertia-Gravity Internal Waves on a Two-Dimensional Shear Flow. Physical Oceanography, [e-journal] 28(4), pp. 363-375. doi:10.22449/1573-160X-2021-4-363-375

DOI: 10.22449/1573-160X-2021-4-363-375

(C) A. A. Slepyshev, 2021

(C) Physical Oceanography, 2021

The problem of vertical exchange remains relevant at the present time, since it is the vertical exchange that ensures the oxygen supply to the deep layers of the sea and the removal of organic material from the deep layers. This is extremely important for the functioning of the ecosystem. According to existing concepts, vertical exchange is provided by small-scale turbulence [1-4]. However, in the pycnocline region turbulence is strongly suppressed by buoyancy forces. 
On the other hand, the pycnocline is a waveguide for internal waves, and it seems relevant to study the contribution of internal waves to vertical exchange. Internal waves propagate mainly by wave trains localized in space [5]. Nonlinear effects during the propagation of trains of internal waves are manifested in the generation of currents averaged on the wave scale $[6,7]$. The vertical velocity component of this current has different signs at the leading and trailing edges of the train, and there is no integral vertical transfer. After the wave train passage, the unperturbed stratification profile is reconstructed. A number of works [8, 9] are devoted to the study of the vertical transfer of momentum by internal waves. Vertical wave momentum fluxes are nonzero with allowance for turbulent viscosity and diffusion [8]. In this case, the wave attenuates [10,11] and the phase shift between the oscillations of vertical and horizontal velocity differs from $\pi / 2$. For inertiagravity internal waves in the presence of a current velocity component transverse to the direction of wave propagation, which depends on the vertical coordinate, the vertical wave momentum flux is nonzero even if turbulent viscosity and diffusion are not taken into account. The point is that the boundary value problem for the amplitude of the vertical velocity of internal waves fixed mode has complex coefficients. This problem was solved analytically in [9] at a constant Brunt - Väisälä frequency and a linear current velocity profile. In this case, the wave propagated perpendicular to the flow. It was obtained that the solution of the boundary value problem (eigenfunction of internal waves) is complex, and the wave frequency is real. When internal waves propagate at an angle to a planeparallel flow with no regard to the Earth's rotation, the wave frequency is real and the eigenfunction is real, since the equation for the vertical velocity amplitude has real coefficients $[12,13]$. It will be obtained below that in a two-dimensional stratified current, taking into account the Earth's rotation, the wave frequency is complex, and the imaginary part of the frequency can be both negative and positive, i.e. both weak attenuation and weak amplification of the wave are possible. In this case, the vertical wave momentum flux and the vertical component of the Stokes drift velocity are nonzero. In [9], the vertical wave momentum flux is not zero, but the vertical component of the Stokes drift velocity is zero, since the wave frequency is real.

Statement of the problem. In the Boussinesq approximation, free internal waves are considered taking into account the Earth's rotation in an infinite basin of constant depth on a two-dimensional current, two horizontal velocity components $U_{0}(z), V_{0}(z)$ of which depend on the vertical coordinate. In the linear approximation, the boundary value problem for the amplitude of internal waves vertical velocity is numerically solved, and the complex frequency of internal waves fixed mode at a constant wavenumber is found. In the second order in wave amplitude, the Stokes drift velocity of liquid particles and vertical wave momentum fluxes are found.

Hydrodynamics equations for wave disturbances have the form

$$
\frac{D u}{D t}-f v+w \frac{d U_{0}}{d z}=-\frac{1}{\rho_{0}(0)} \frac{\partial P}{\partial x},
$$




$$
\begin{gathered}
\frac{D v}{D t}+f u+w \frac{d V_{0}}{d z}=-\frac{1}{\rho_{0}(0)} \frac{\partial P}{\partial y}, \\
\frac{D w}{D t}=-\frac{1}{\rho_{0}(0)} \frac{\partial P}{\partial z}-\frac{g \rho}{\rho_{0}(0)}, \\
\frac{D \rho}{D t}=-w \frac{d \rho_{0}}{d z}, \\
\frac{\partial u}{\partial x}+\frac{\partial v}{\partial y}+\frac{\partial w}{\partial z}=0,
\end{gathered}
$$

where $u, v, w$ are two horizontal and vertical components of current wave velocity, respectively; $\rho, P$ are wave disturbances of density and pressure; $\rho_{0}(z)$ is an average density profile; $x, y, z$ are two horizontal and one vertical coordinates, $z$ axis is directed upwards; $f$ is the Coriolis parameter; $U_{0}(z), V_{0}(z)$ are two velocity components of mean current; Action of $\frac{D}{D t}$ operator is expanded by the formula $\frac{D}{D t}=\frac{\partial}{\partial t}+\left(u+U_{0}\right) \frac{\partial}{\partial x}+\left(v+V_{0}\right) \frac{\partial}{\partial y}+w \frac{\partial}{\partial z}$. Using geostrophic relations from [14], we are to estimate the horizontal scales of mean density variation: $L_{x}=\rho_{0} / \frac{\partial \rho_{0}}{\partial x}=g /\left(f \max \left|\frac{\partial V_{0}}{\partial z}\right|\right), L_{y}=\rho_{0} / \frac{\partial \rho_{0}}{\partial y}=g /\left(f \max \left|\frac{\partial U_{0}}{\partial z}\right|\right)$.

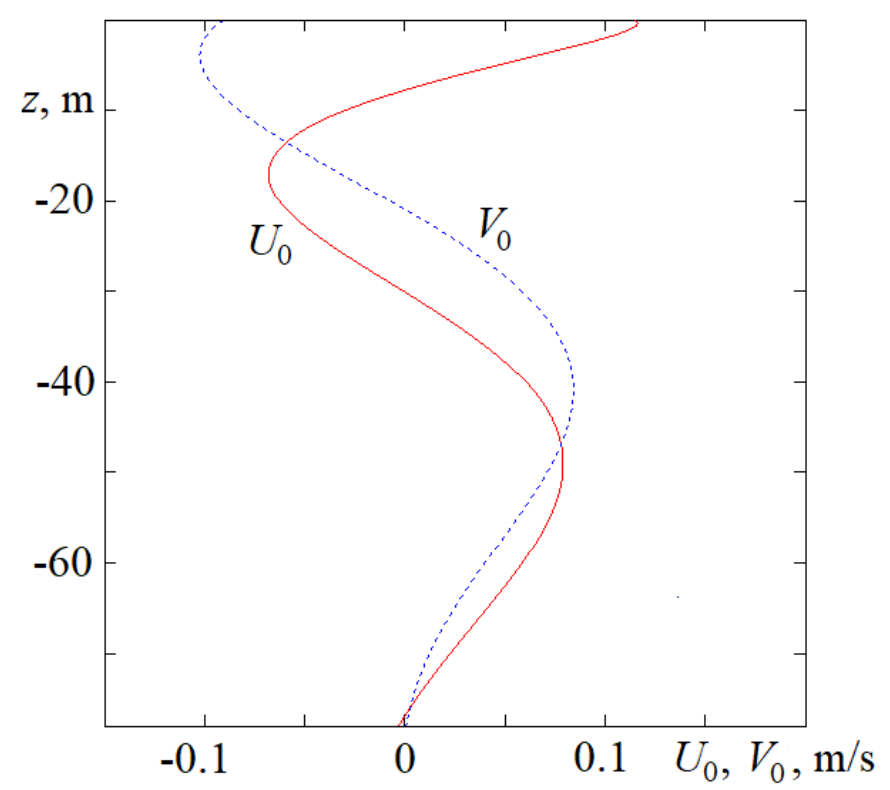

F i g. 1. Vertical profiles of the current velocity components $U_{0}, V_{0}$

The vertical profiles of the current velocity components are demonstrated in Fig. 1. The maximum values of the modulus of vertical gradients of current velocity components $U_{0}, V_{0}$ are 0.067 and $0.011 \mathrm{l} / \mathrm{s}$, respectively. The horizontal PHYSICAL OCEANOGRAPHY VOL. 28 ISS. 4 (2021) 
scales of density variation are equal to $L_{x}=8.283 \cdot 10^{6} \mathrm{~m}, L_{y}=1.398 \cdot 10^{6} \mathrm{~m}$, respectively, and they are much larger than the wavelength, therefore, the horizontal density variation is neglected.

The boundary condition on the sea surface $z=0$ is the "rigid lid" condition that filters internal waves from the surface waves [15]:

$$
w(0)=0 .
$$

The boundary condition at the bottom is impermeability condition:

$$
w(-H)=0 \text {, }
$$

where $H$ is a sea depth.

Linear approximation. We seek solutions of linear approximation in the following form:

$$
\begin{gathered}
u_{1}=u_{10}(z) A \mathrm{e}^{i \theta}+\text { c.c. }, \quad v_{1}=v_{10}(z) A \mathrm{e}^{i \theta}+\text { c.c. }, \quad w_{1}=w_{10}(z) A \mathrm{e}^{i \theta}+\text { c.c. }, \\
P_{1}=P_{10}(z) A \mathrm{e}^{i \theta}+\text { c.c. }, \quad \rho_{1}=\rho_{10}(z) A \mathrm{e}^{i \theta}+\text { c.c. },
\end{gathered}
$$

where c.c. are complex conjugate terms; $A$ is an amplitude factor; $\theta$ is a wave phase $(\partial \theta / \partial x=k, \partial \theta / \partial t=-\omega, k$ is a horizontal wavenumber, $\omega$ is a wave frequency). It is assumed that the wave propagates along $x$ axis.

After substituting (8) into system (1) - (5), the amplitude functions $u_{10}, v_{10}, \rho_{10}, P_{10}$ are linked with $w_{10}$ :

$$
\begin{gathered}
u_{10}=\frac{i}{k} \frac{d w_{10}}{d z}, \quad \Omega=\omega-k \cdot U_{0}, \\
\frac{P_{10}}{\rho_{0}(0)}=\frac{i}{k}\left[\frac{\Omega}{k} \frac{d w_{10}}{d z}+\frac{d U_{0}}{d z} w_{10}+\frac{f}{\Omega}\left(i \frac{d V_{0}}{d z} w_{10}-\frac{f}{k} \frac{d w_{10}}{d z}\right)\right], \\
\rho_{10}=-\frac{i}{\Omega} w_{10} \frac{d \rho_{0}}{d z}, \quad v_{10}=\frac{1}{\Omega}\left(\frac{f}{k} \frac{d w_{10}}{d z}-i w_{10} \frac{d V_{0}}{d z}\right) .
\end{gathered}
$$

Function $w_{10}$ satisfies the equation

$$
\begin{aligned}
& \frac{d^{2} w_{10}}{d z^{2}}+k\left[\frac{i f \frac{d V_{0}}{d z}}{\Omega^{2}-f^{2}}-\frac{f^{2} \frac{d U_{0}}{d z}}{\Omega\left(\Omega^{2}-f^{2}\right)}\right] \frac{d w_{10}}{d z}+ \\
& +k w_{10}\left[\frac{k\left(N^{2}-\Omega^{2}\right)+\Omega \frac{d^{2} U_{0}}{d z^{2}}+i f \frac{d^{2} V_{0}}{d z^{2}}}{\Omega^{2}-f^{2}}+\frac{i f k \frac{d U_{0}}{d z} \frac{d V_{0}}{d z}}{\Omega\left(\Omega^{2}-f^{2}\right)}\right]=0,
\end{aligned}
$$


where $N^{2}=-\frac{g}{\rho_{0}(0)} \frac{d \rho_{0}}{d z}$ is a square of Brunt - Väisälä frequency.

Boundary conditions for $w_{10}$ :

$$
\begin{aligned}
& \text { at } Z=0 \quad w_{10}=0, \\
& \text { at } Z=-H \quad w_{10}=0 .
\end{aligned}
$$

Boundary value problem (12)-(14) was solved analytically in [9] at a constant Brunt - Väisälä frequency and a linear velocity profile of a planeparallel mean current. The wave propagated perpendicular to the flow. It was found that the eigenfunction - the solution to this boundary value problem - is complex, and the wave frequency is real. Below, while carrying out numerical calculations, it will be shown that the wave frequency is complex in a two-dimensional current.

In [16], equation (12) was solved by the perturbation method by expanding the solution in a series in a small parameter $\varepsilon=V_{0}^{*} /\left(H \omega_{*}\right)$, where $V_{0}^{*}$ is characteristic value of current velocity and $\omega_{*}$ is the characteristic wave frequency. However, this parameter is not always small and is the applicability of the method is limited. Therefore, in the present work, the implicit Adams scheme of the third order of accuracy is used for the numerical solution of equation (12) for real profiles of Brunt - Väisälä frequency and current velocity.

Boundary value problem (12) - (14) was solved analytically in [12, 13, 17] for a plane-parallel flux with a linear current velocity profile at $f=0, N=$ const in case when the wave propagates at an arbitrary angle to the flow. A dispersion equation containing modified Bessel functions is obtained. In [17], only the first three terms were left in the expansion of the Bessel functions in a series; therefore, the imaginary part of the phase velocity is greater than zero even at the Richarson number $\mathrm{Ri}=\frac{N^{2}}{\left(\frac{d U}{d z}\right)^{2}}>\frac{1}{4}$, which contradicts the Miles criterion $[18,19]$ of a plane-

parallel flow hydrodynamic stability. However, in [12, 13], the same dispersion equation was solved numerically and asymptotically, and no contradiction to Miles criterion of hydrodynamic stability was found. In [20], for an arbitrary planeparallel flow with a continuous velocity profile at $N=$ const, no contradiction to the Miles criterion was also revealed.

It is of interest to compare the numerical solution of boundary value problem (12) - (14) according to the implicit Adams scheme of the third order of accuracy with the analytical solution [9] for a plane-parallel flow with a linear flow velocity profile at $N=$ const when the wave propagates perpendicular to the flow. For the first mode 15-minute internal waves with $\omega=0.00698132 \mathrm{rad} / \mathrm{s}$ frequency, a numerical calculation provides the wavenumber value $k=0.0418831513 \mathrm{rad} / \mathrm{m}$. If this value is substituted into the formula for the frequency of the analytical solution (20) from [9], then the frequency value is $\omega=0.00698133 \mathrm{rad} / \mathrm{s}$. The frequency difference from numerical solution is $10^{-4} \%$. A similar calculation 
for the second mode gives a discrepancy between the numerical and analytical solutions for the frequency in $3 \cdot 10^{-4} \%$.

Now we are to compare the numerical solution of the boundary value problem (12) - (14) according to the implicit Adams scheme of the third order of accuracy with the analytical solution from [12] for a plane-parallel flow with a linear velocity profile at $f=0, N=$ const, when the wave propagates at an arbitrary angle to the flow. For this purpose, we use the second model of the flow velocity from [12], when the specified velocity is zero at the bottom. Wave frequency $\omega$ in [12] (normalized to the Brunt - Väisälä frequency) was taken equal to 0.54 in dimensionless variables. Let $\mu, v$ be the projections of the dimensionless wave vector (normalized to $\pi / H$ ) onto the directions along and across the flow. Then, for $v=0.5$ the numerical solution of the boundary value problem (4) in [12] according to the implicit Adams scheme of the third order of accuracy provides the value $\mu=0.1742$ for the first mode, which corresponds to both the numerical and asymptotic solutions of the dispersion equation (5) in [12].

Nonlinear effects. The Stokes drift velocity of liquid particles is determined by the formula from [21]:

$$
\overrightarrow{\mathrm{u}}_{s}=\overline{\int_{0}^{t}(\overrightarrow{\mathrm{u}} d \tau \nabla) \overrightarrow{\mathrm{u}}},
$$

where $\overrightarrow{\mathrm{u}}$ is a field of Euler wave velocities, the bar above means averaging over the wave period. The horizontal velocity component of the Stokes drift, directed along the wave vector, up to terms quadratic in the wave amplitude, has the form

$$
u_{s}=\frac{A_{1} A_{1}^{*}}{k}\left[\frac{1}{\omega} \frac{d}{d z}\left(w_{10} \frac{d w_{10}^{*}}{d z}\right)+\text { c.c. }\right] \text {, }
$$

where $A_{1}=A \exp (\delta \omega \cdot t), \delta \omega=\operatorname{Im}(\omega)$ is an imaginary part of wave frequency.

The horizontal component of the Stokes drift velocity transverse to the wave propagation direction is determined by the formula

$$
\begin{aligned}
& v_{s}=A_{1} A_{1}^{*}\left[\frac{1}{\omega \Omega^{*} k} \frac{d w_{10}}{d z}\left(\text { if } \frac{d w_{10}^{*}}{d z}-k \frac{d V_{0}}{d z} w_{10}^{*}\right)+\text { c.c. }\right]- \\
& -A_{1} A_{1}^{*}\left\{\frac{w_{10}^{*}}{\omega^{*} \Omega^{2}}\left[\Omega\left(\frac{d w_{10}}{d z} \frac{d V_{0}}{d z}+\frac{i f}{k} \frac{d^{2} w_{10}}{d z^{2}}+w_{10} \frac{d^{2} V_{0}}{d z^{2}}\right)+k \frac{d U_{0}}{d z}\left(w_{10} \frac{d V_{0}}{d z}+\frac{i f}{k} \frac{d w_{10}}{d z}\right)\right]+\text { c.c. }\right\} .
\end{aligned}
$$

In the presence of an average current, for which the velocity component $V_{0}$ transverse to the wave propagation direction depends on the vertical coordinate, the value $v_{s}$ is nonzero. The vertical component of the Stokes drift velocity is proportional to the imaginary part of the frequency and is determined by the formula

$$
w_{s}=i A_{1} A_{1}^{*}\left(\frac{1}{\omega}-\frac{1}{\omega^{*}}\right) \frac{d}{d z}\left(w_{10} w_{10}^{*}\right) .
$$


We find vertical wave momentum fluxes $\overline{u w}, \overline{v w}$ taking into account (8), (9), (11):

$$
\begin{gathered}
\overline{u w}=\frac{i}{k}\left|A_{1}^{2}\right|\left(w_{10}^{*} \frac{d w_{10}}{d z}-w_{10} \frac{d w_{10}^{*}}{d z}\right), \\
\overline{v w}=\left|A_{1}^{2}\right|\left[\frac{i w_{10} w_{10}^{*}}{\Omega \Omega^{*}}\left(\Omega-\Omega^{*}\right) \frac{d V_{0}}{d z}+\frac{f}{\Omega \Omega^{*} k}\left(\Omega^{*} w_{10}^{*} \frac{d w_{10}}{d z}+\Omega w_{10} \frac{d w_{10}^{*}}{d z}\right)\right] .
\end{gathered}
$$

The vertical wave flux of momentum $\overline{v w}$ in formula (20) is nonzero and in the absence of flow, the flux of momentum $\overline{u w}$ in formula (19) is nonzero only in the presence of an average current, for which the velocity component $V_{0}$ transverse to the direction of wave propagation depends on the vertical coordinate. In the absence of the Earth's rotation at $f=0$, the wave momentum fluxes are equal to zero.

Calculation results. We will analyze the vertical momentum fluxes for internal waves and the Stokes drift velocity using the results of the third stage of the $44^{\text {th }}$ cruise of $\mathrm{R} / \mathrm{V}$ Mikhail Lomonosov in the area of the Black Sea northwestern shelf ${ }^{1}$. The profiles of two components of the mean current velocity are given in Fig. 1, the Brunt - Väisälä frequencies are depicted in Fig. 2.

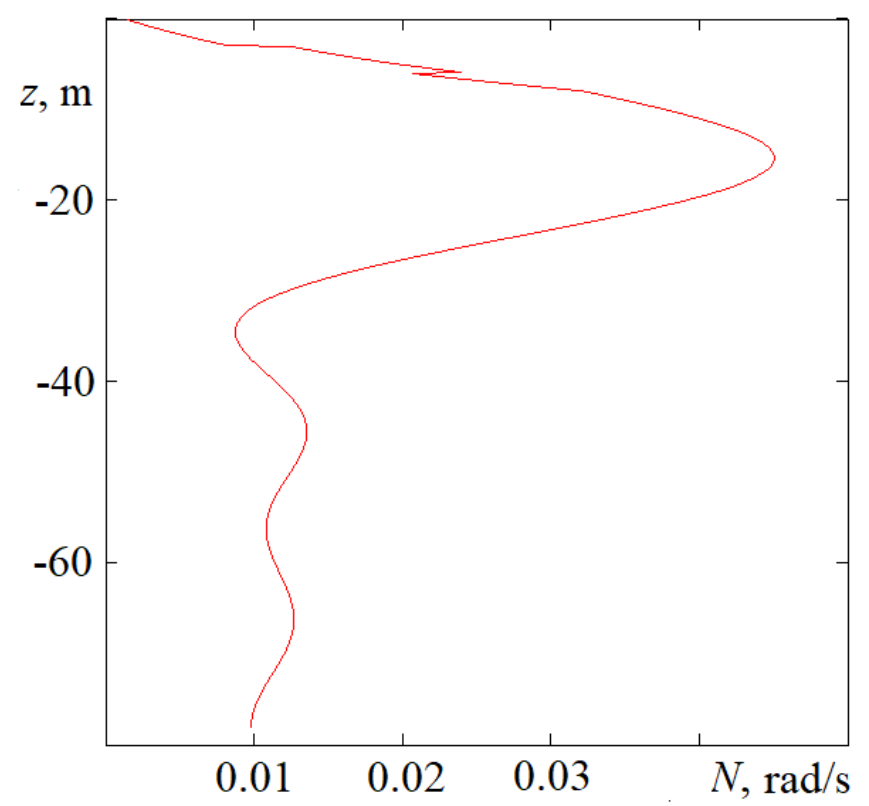

F i g. 2. Vertical profile of the Brunt - Väisälä frequency

${ }^{1}$ MHI AS USSR, 1985. Report on the Work in the $44^{\text {th }}$ Cruise ( $3^{\text {rd }}$ Stage) of R/V Mikhail Lomonosov, August, 7 - September, 15. Sevastopol: MHI AS USSR. Vol. 1, 135 p. (in Russian). 
Boundary value problem (12) - (14) of determining the mode vertical structure is solved numerically according to the implicit Adams scheme of the third order of accuracy. The wave frequency at a fixed wavenumber is found by the shooting method from the need to fulfill the boundary conditions (13), (14). The eigenfunction (the solution to the boundary value problem (12) - (14)) is complex, therefore the vertical wave flux of momentum $\overline{u w}$ (18) is nonzero. The dependences of real and imaginary wave frequency parts on the wave number for the first two modes are shown in Fig. 3, 4. In Fig. 3 the dispersion curves of the first two modes are given, $\omega_{0}=\operatorname{Re}(\omega)$ is the real part of the frequency.

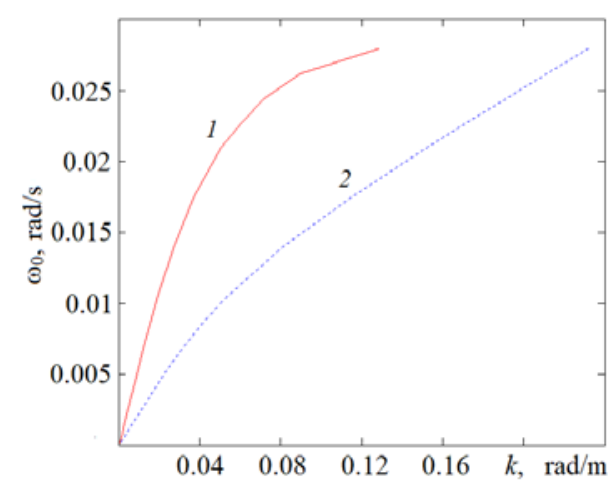

F i g. 3. Dependence of the wave frequency real part on the wave number for the first 1 and second 2 modes

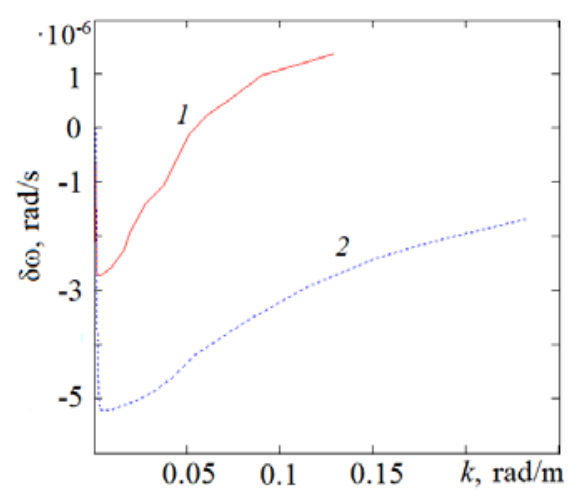

F i g. 4. Dependence of the wave frequency imaginary part on the wave number for the first 1 and second 2 modes

In Fig. 4, the imaginary part of frequency for the first mode is negative at $k<0.055 \mathrm{rad} / \mathrm{m}$ and positive at $k>0.055 \mathrm{rad} / \mathrm{m}$ are demonstrated. In absolute value, the imaginary part of the frequency is three to four orders of magnitude less than the real part of the frequency. Thus, the first mode has a weak attenuation in the low-frequency region at $k<0.055 \mathrm{rad} / \mathrm{m}$, and weak wave amplification in the high-frequency region at $k>0.055 \mathrm{rad} / \mathrm{m}$. In the second mode, the imaginary part of the frequency is always negative, i.e. the wave attenuates weakly. In the first mode attenuation region, the attenuation decrement for the second mode is greater in absolute value.

It is of interest to study the vertical momentum fluxes and the Stokes drift velocity for the first mode waves with different signs of the imaginary part of the frequency, for example, for internal waves with frequencies of 10 and $16 \mathrm{cph}$ (meaning the real part of the frequency). For a wave of the first mode with a $10 \mathrm{cph}$ frequency, the imaginary part of $\delta \omega=-8.378 \cdot 10^{-7} \mathrm{rad} / \mathrm{s}$ is negative. For a wave of the same mode with $16 \mathrm{cph}$ frequency, the imaginary part of $\delta \omega=1.379 \cdot 10^{-6} \mathrm{rad} / \mathrm{s}$ is positive. Therefore, further we will compare the vertical wave momentum fluxes and the Stokes drift velocity for these waves. In Fig. 5 the profiles of vertical momentum fluxes $\overline{u w}$ for internal waves of the first mode with $0.5 \mathrm{~m}$ amplitude and frequencies of $10 \mathrm{cph}$ (red line) and $16 \mathrm{cph}$ (blue line), as well as turbulent flux $\overline{u^{\prime} w^{\prime}}$ (green line) are given. 
The turbulent momentum flux is determined by the formula $\overline{u^{\prime} w^{\prime}}=-K_{z} \frac{d U_{0}}{d z}$, the coefficient of vertical turbulent exchange - by the formula $K_{z} \cong 0.93 \cdot 10^{-4} N_{c}^{-1}$ $\mathrm{m}^{2} / \mathrm{s}$ ( $N_{c}$ corresponds to the Brunt - Väisälä frequency in cph) [22, 23]. In general, with the exception of 15-25 m depth interval, the turbulent flux prevails over the wave flux. The wave momentum flux for waves with $10 \mathrm{cph}$ frequency prevails over the flux for waves with a frequency of $16 \mathrm{cph}$ in the depth interval of $15-40 \mathrm{~m}$. In the upper 15-meter layer, there is a slight predominance of the flux for waves with a frequency of $16 \mathrm{cph}$. Deeper than $40 \mathrm{~m}$, wave fluxes are comparable in magnitude.

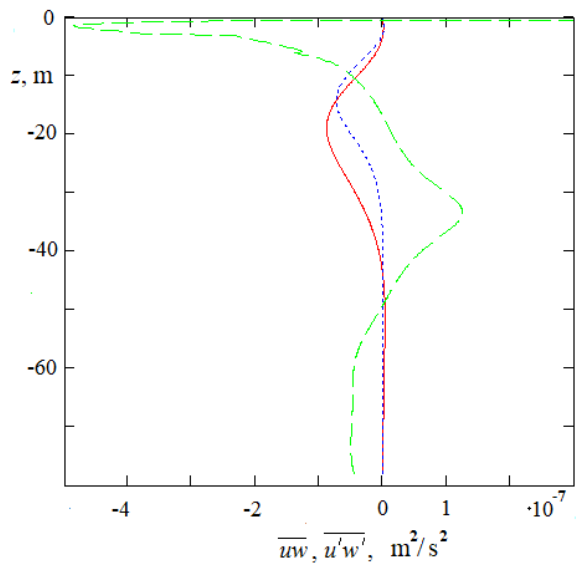

F i g. 5. Profiles of the wave $\overline{u w}$ and turbulent $\overline{u^{\prime} w^{\prime}}$ vertical momentum fluxes

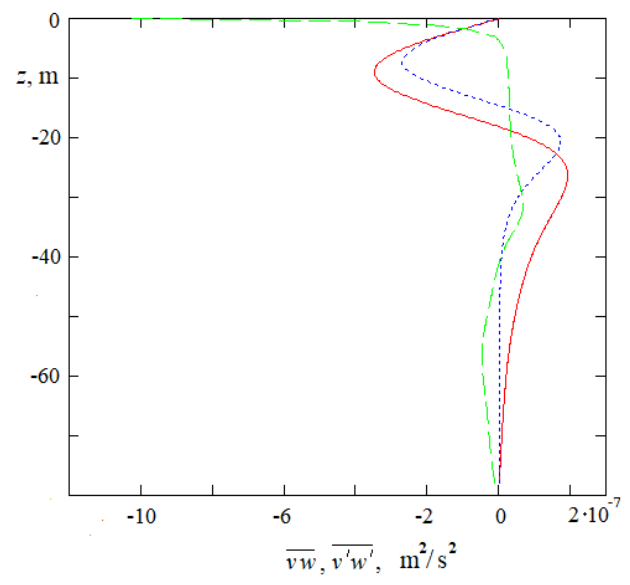

F i g. 6. Profiles of the wave $\overline{v w}$ and turbulent $\overline{v^{\prime} w^{\prime}}$ vertical momentum fluxes

In Fig. 6 the profiles of vertical fluxes of momentum $\overline{v w}$ for internal waves of the first mode with $10 \mathrm{cph}$ (red line) and $16 \mathrm{cph}$ (blue line) frequencies, as well as turbulent flux $\overline{v^{\prime} w^{\prime}}$ (green line) are demonstrated.

The turbulent momentum flux is determined by the formula $\overline{v^{\prime} w^{\prime}}=-K_{z} \frac{d V_{0}}{d z}$. The dominance of turbulent flux over the wave flux is already absent here. In 2.5$40 \mathrm{~m}$ depth interval of the waves with $10 \mathrm{cph}$ frequency vertical momentum flux $\overline{v w}$ exceeds the turbulent one in absolute value. For the waves with $16 \mathrm{cph}$ frequency, the vertical momentum flux $\overline{v w}$ is less than for the waves with $10 \mathrm{cph}$ frequency.

It is of interest to compare $\overline{u w}$ and $\overline{v w}$ fluxes with each other. In Fig. 7 the profiles of these fluxes for the first mode waves with $10 \mathrm{cph}$ frequency are represented. In general, $\overline{u w}$ flux (red line) is noticeably inferior to $\overline{v w}$ flux (blue line). 
Now we are to compare the Stokes drift velocity for internal waves of the first mode with 10 and $16 \mathrm{cph}$ frequencies. The vertical profiles of the Stokes drift velocity component along the wave propagation direction (formula (16)) are demonstrated in Fig. 8 for the waves with $10 \mathrm{cph}$ (red line) and $16 \mathrm{cph}$ frequency (blue line). The structure of the profiles of the Stokes drift velocity longitudinal component is very similar, only in the upper 10-m layer and deeper than $30 \mathrm{~m}$ the Stokes drift velocity is higher for the waves with 10 cph frequency.

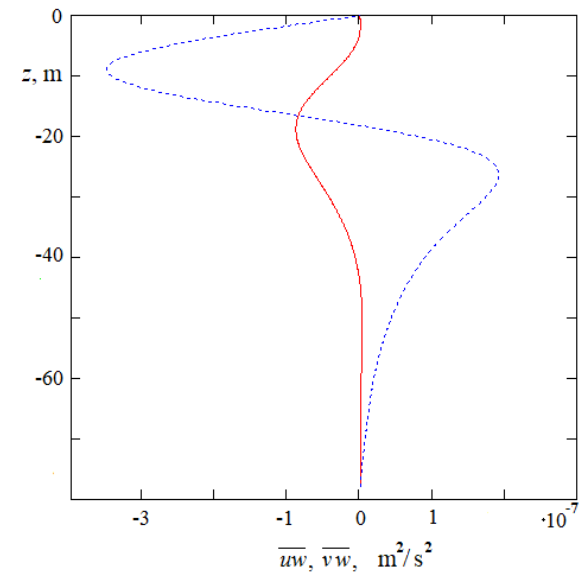

F i g. 7. Profiles of the wave $\overline{u w}, \overline{v w}$ vertical momentum fluxes

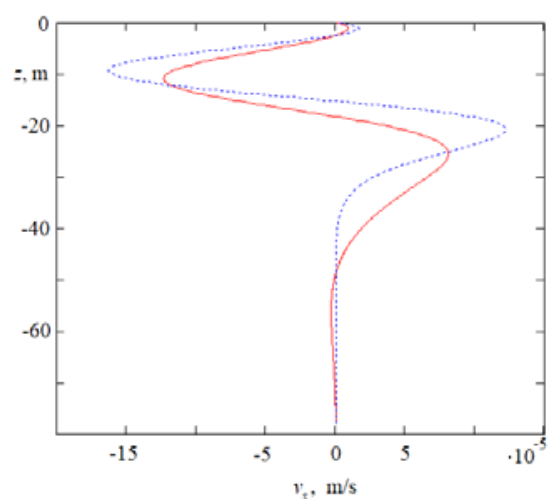

F i g. 9. Profiles of the Stokes drift velocity component transverse to the wave propagation direction

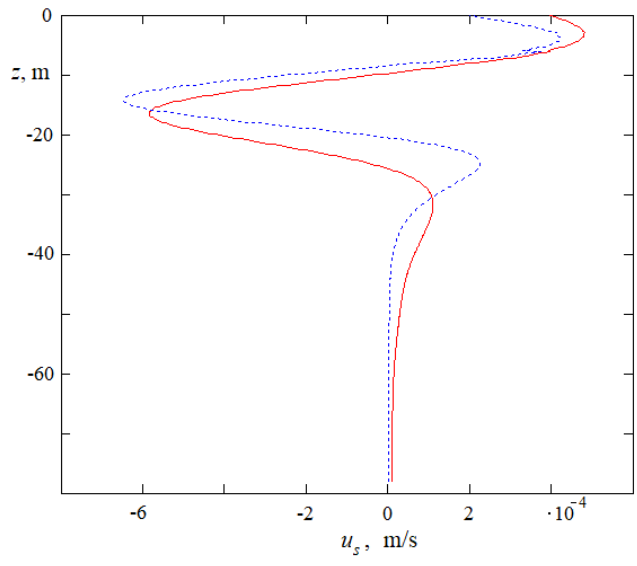

F i g. 8. Vertical profiles of the longitudinal component of the Stokes drift velocity

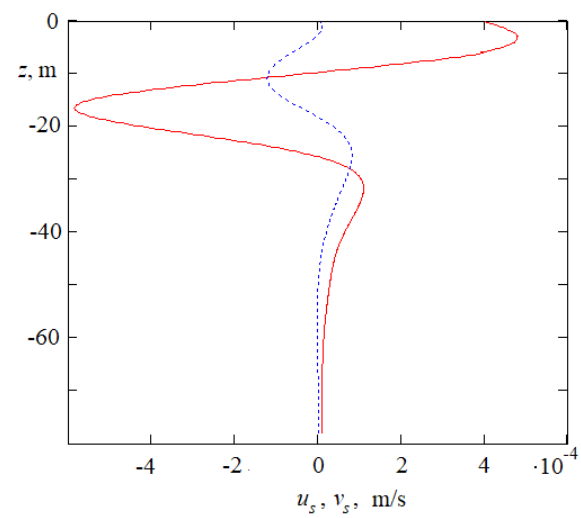

F i g. 10. Vertical profiles of two components of the Stokes drift horizontal velocity

The vertical profiles of the Stokes drift velocity component transverse to the direction of wave propagation (formula (17)) for waves with $10 \mathrm{cph}$ (red line) and $16 \mathrm{cph}$ (blue line) frequencies are demonstrated in Fig. 9. Here, in the upper 25-m layer, the moduli of the maximum and minimum values of this velocity component for the waves with $16 \mathrm{cph}$ frequency is higher, while deeper than $25 \mathrm{~m}$ 
and up to $48 \mathrm{~m}$ depth, the Stokes drift velocity prevails for the waves with $10 \mathrm{cph}$ frequency.

It is of interest to compare the Stokes drift velocities along and across the direction of wave propagation. In Fig. 10 the vertical profiles of these velocity components for the waves with $10 \mathrm{cph}$ period are presented. The Stokes drift velocity $u_{s}$ along the direction of wave propagation (red line) noticeably exceeds the transverse velocity $v_{s}$ (blue line).

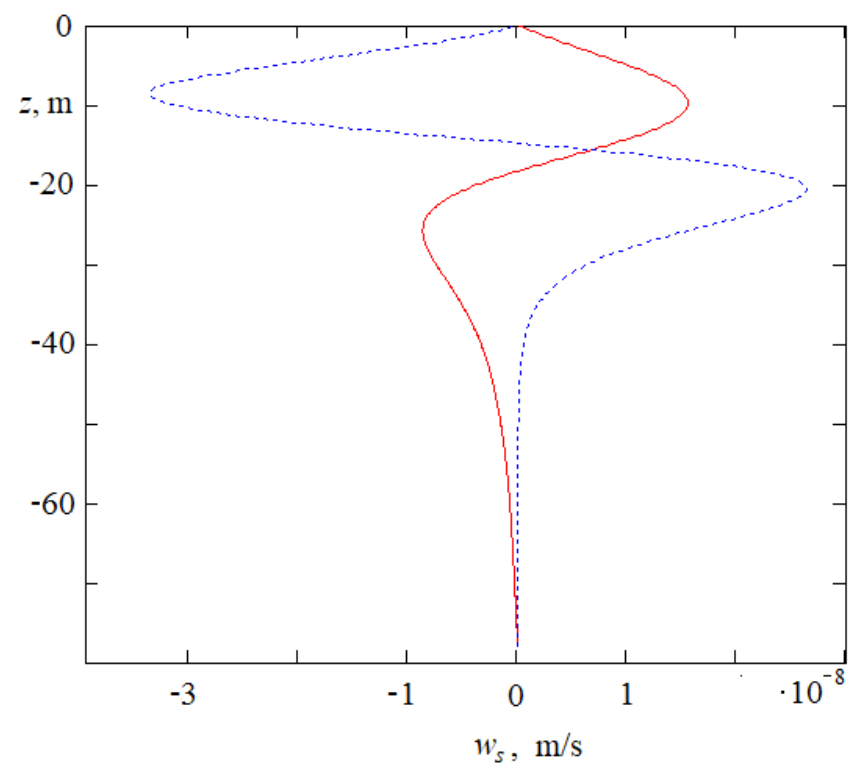

F i g. 11. Profiles of the vertical component of the Stokes drift velocity

Now we are to compare the vertical component of the Stokes drift velocity (formula (18)) for waves with of 10 and $16 \mathrm{cph}$ frequencies. In Fig. 11 the maximum (in modulus) velocity prevails for the waves with frequency $16 \mathrm{cph}$ (blue line). The directions of vertical component of Stokes drift velocity for the waves with 10 and $16 \mathrm{cph}$ frequencies are opposite, since it is proportional to the imaginary part of frequency and differs in sign. The magnitude of the vertical component of this velocity is very small, four orders of magnitude less than the velocity along the direction of wave propagation. However, it can make a significant contribution to the vertical heat and salt transfer, as well as to the vertical fine structure generation [24].

\section{Conclusions}

1. Vertical momentum fluxes of wave $\overline{u w}, \overline{v w}$ of internal waves at twodimensional shear flow with regard to the Earth rotation differ from zero and can be comparable or exceed corresponding turbulent fluxes.

2. The imaginary part of wave frequency differs from zero and is negative for the second mode - weak wave attenuation takes place. In the first mode 
the attenuation occurs in the low frequency area at $k<0.055 \mathrm{rad} / \mathrm{m}$, at $k>0.055 \mathrm{rad} / \mathrm{m}$ a weak wave amplification takes place.

3. The Stokes drift velocity along the direction of wave propagation noticeably exceeds transverse velocity.

4. The vertical components of the Stokes drift velocity of the first mode waves with 10 and $16 \mathrm{cph}$ frequencies has different signs due to the fact that it is proportional to the imaginary part of frequency.

\section{REFERENCES}

1. Panteleev, N.A., Okhotnikov, I.N. and Slepyshev, A.A., 1993. Small-Scale Structure and Dynamics of the Ocean. Kiev: Naukova Dumka, 195 p. (in Russian).

2. Samodurov, A.S., Chukharev, A.M., Zubov, A.G. and Pavlenko, O.I., 2015. StructureFormation and Vertical Turbulent Exchange in the Coastal Area of the Sevastopol Region. Physical Oceanography, (6), pp. 3-14. doi:10.22449/1573-160X-2015-6-3-14

3. Wunsch, C. and Ferrari, R., 2004. Vertical Mixing, Energy, and the General Circulation of the Oceans. Annual Review of Fluid Mechanics, 36, pp. 281-314. https://doi.org/10.1146/annurev.fluid.36.050802.122121

4. Vasil'ev, O.F., Voropaeva, O.F., Kurbatskii, A.F., 2011. Turbulent Mixing in Stably Stratified Flows of the Enviroment: The Current State of the Problem (Review). Izvestiya, Atmospheric and Ocean Physics, 47(3), pp. 265-280.

5. Bulatov, V.V. and Vladimirov, Yu.V., 2015. [Waves in Stratified Media]. Moscow: Nauka, 735 p. (in Russian).

6. Borisenko, Yu.D., Voronovich, A.G., Leonov, A.I. and Miropolsky, Yu.Z., 1976. On the Theory of Non-Stationary Weak Nonlinear Internal Waves in Stratified Fluid. Izvestiya AN SSSR, Fizika Atmosfery i Okeana, 12(3), pp. 293-301 (in Russian).

7. Grimshaw, R., 1977. The Modulation of an Internal Gravity-Wave Packet, and the Resonance with the Mean Motion. Studies in Applied Mathematics, 56(3), pp. 241-266. doi:10.1002/sapm1977563241

8. Slepyshev, A.A., 2016. Vertical Momentum Transfer by Internal Waves when Eddy Viscosity and Diffusion are taken into Account. Izvestiya, Atmospheric and Oceanic Physics, 52(3), pp. 301-308. doi:10.1134/S0001433816030117

9. Slepyshev, A.A. and Laktionova, N.V., 2019. Vertical Transport of Momentum by Internal Waves in a Shear Current. Izvestiya, Atmospheric and Oceanic Physics, 55(6), pp. 662-668. https://doi.org/10.1134/S0001433819060148

10. LeBlond, P.H. and Mysak, L.A., 1978. Waves in the Ocean. Amsterdam: Elsevier Scientific Publishing Company, $602 \mathrm{p}$.

11. LeBlond, P.H., 1966. On the Damping of Internal Gravity Waves in a Continuously Stratified Ocean. Journal of Fluid Mechanics, 25(1), pp. 121-142. doi:10.1017/S0022112066000089

12. Bulatov, V.V. and Vladimirov, Yu.V., 2020. Dynamics of Internal Gravity Waves in the Ocean with Shear Flows. Russian Journal of Earth Sciences, 20(4), ES4004. doi:10.2205/2020ES000732

13. Bulatov, V. and Vladimirov, Yu., 2020. Analytical Approximations of Dispersion Relations for Internal Gravity Waves Equation with Shear Flows. Symmetry, 12(11), 1865. doi:10.3390/sym12111865

14. Kamenkovich, V.M., 1977. Fundamentals of Ocean Dynamics. Amsterdam: Elsevier Scientific Publishing Company, 249 p.

15. Miropol'sky, Yu.Z., 2013. Dynamics of Internal Gravity Waves in the Ocean. Cham: Springer Science \& Business Media, 406 p.

16. Vorotnikov, D.I. and Slepyshev, A.A., 2018. Vertical Momentum Fluxes Induced by Weakly Nonlinear Internal Waves on the Shelf. Fluid Dynamics, 53(1), pp. 21-33. https://doi.org/10.1134/S0015462818010160 
17. Gavril'eva, A.A., Gubarev, Yu.G. and Lebedev, M.P., 2019. The Miles Theorem and the First Boundary Value Problem for the Taylor-Goldstein Equation. Journal of Applied and Industrial Mathematics, 13(3), pp. 460-471. doi:10.1134/S1990478919030074

18. Miles, J.W., 1961. On the Stability of Heterogeneous Shear Flows. Journal of Fluid Mechanics, 10(4), pp. 496-508. doi:10.1017/S0022112061000305

19. Howard, L.N., 1961. Note on a Paper of John Miles. Journal of Fluid Mechanics, 10(4), pp. 509-512. doi:10.1017/S0022112061000317

20. Banks, W.H.H., Drazin, P.G. and Zaturska, M.B., 1976. On the Normal Modes of Parallel Flow of Inviscid Stratified Fluid. Journal of Fluid Mechanics, 75(1), pp. 149-171. doi:10.1017/S0022112076000153

21. Longuet-Higgins, M.S., 1969. On the Transport of Mass by Time-Varying Ocean Current. Deep Sea Research and Oceanographic Abstracts, 16(5), pp. 431-447. doi.org/10.1016/00117471(69)90031-X

22. Ivanov, V.A., Samodurov, A.S., Chukharev, A.M. and Nosova, A.V., 2008. [Intensification of Vertical Turbulent Exchange in the Areas of the Interface of the Shelf and the Continental Slope in the Black Sea]. Dopovidi NAS of Ukraine = Reports of the National Academy of Sciences of Ukraine, (6), pp. 108-112.

23. Samodurov, A.S., 2016. Complementarity of Different Approaches for Assessing Vertical Turbulent Exchange Intensity in Natural Stratified Basins. Physical Oceanography, (6), pp. 32-42. doi:10.22449/1573-160X-2016-6-32-42

24. Slepyshev, A.A. and Vorotnikov, D.I., 2018. Vertical Mass Transport by Weakly Nonlinear Inertia-Gravity Internal Waves. In: V. Karev, D. Klimov, K. Pokazeev, eds., 2018. Physical and Mathematical Modeling of Earth and Environment Processes. PMMEEP 2017. Springer, Cham, pp. 99-111. doi.org/10.1007/978-3-319-77788-7_12

About the author:

Aleksandr A. Slepyshev, Leading Research Associate, Marine Hydrophysical Institute of RAS (2 Kapitanskaya St., Sevastopol, 299011, Russian Federation), Dr. Sci. (Phys.-Math.), ResearcherID: V-6948-2017, ORCID ID: 0000-0002-9259-7558, IstinaResearcherID (IRID): 3614585, slep55@mail.ru

The author has read and approved the final manuscript.

The author declares that he has no conflict of interest. 